\title{
Aero-Structural Design Optimization of Adaptive Shock Control Bumps
}

\author{
E. Jinks*, M. Santer ${ }^{\dagger}$ and P. Bruce ${ }^{\ddagger}$
}

\begin{abstract}
Shock control bumps (SCB) are a transonic flow control device that aim to reduce the overall drag due to a normal shock on a typical passenger jet at cruise. The concept of adaptive SCB which can be deployed for best use are investigated through an aero-structural design tool that produces optimal geometries. The optimizer uses a surface based performance metric to highlight the importance of the flow quality around the SCB as well as including a structural element that is required to provide the necessary flexibility to deform. The performance metric produces the target pressure distribution and successfully smears the shock. It is found that the structural constraint does not inhibit bump height and global airfoil performance is not significantly affected, $\mathrm{L} / \mathrm{D}$ varies $<0.6 \%$. The aerodynamic pressure loading can be utilised to produce a new family of SCB geometries that are unachievable with mechanical actuation alone. The study shows that adaptive SCB that exploit the naturally occurring pressure field around an airfoil in a passive way are a feasible technology to mitigate the poor off-design performance of static SCB.
\end{abstract}

\section{Nomenclature}

$\begin{array}{ll}\alpha & \text { Angle of attack }\left({ }^{\circ}\right) \\ \delta & \text { Actuation height }(\mathrm{mm}) \\ \nu & \text { Poisson's ratio } \\ \sigma & \text { Material stress }(\mathrm{Pa}) \\ \sigma_{y} & \text { Material yield stress }(\mathrm{Pa}) \\ \xi & \text { Local coordinates } \\ B & \text { Optimization: boundary location }(\mathrm{x} / \mathrm{c}) \\ c & \text { Airfoil chord }(\mathrm{m})\end{array}$

$\begin{array}{ll}E & \text { Young's modulus (Pa) } \\ i & \text { Number of individuals } \\ k & \text { Design stress safety factor } \\ l_{b} & \text { Bump length (m) } \\ M & \text { Optimization: objective function } \\ x, y & \text { Spatial coordinates (m) } \\ C_{D} & \text { Drag coefficient } \\ C_{L} & \text { Lift coefficient } \\ C_{p} & \text { Pressure coefficient } \\ \mathrm{Ma} & \text { Mach number } \\ \mathrm{Re} & \text { Reynolds number }\end{array}$

\section{Introduction}

This paper considers the design of a morphing device for shock control. Many directives are currently in place to improve the efficiency, and reduce the environmental impact of transport aircraft. The Vision 2020 directive, issued by the European commission, targets transport aircraft of the year 2020 to achieve a $50 \%$ cut in carbon dioxide emissions per passenger per kilometre and an $80 \%$ reduction in nitrous oxide emissions. ${ }^{1}$ Refining current designs is reaching a plateau in terms of improving aircraft aerodynamics, therefore engineers are looking to new methods to try to achieve these goals. A limiting factor on the efficiency and flight speed of many commercial aircraft is the presence of wave drag. A typical transonic passenger aircraft cruises at Mach 0.8 where the flow over the upper wing surface is accelerated to supersonic velocities which in turn leads to the formation of a normal shock. The total pressure loss across this shockwave forms a significant contribution to wave drag. This type of drag increases significantly as the aircraft accelerates beyond the design Mach number.

Wave drag occurs due to the presence of shockwaves. Accelerating the flow to supersonic speeds lower the static pressure on the upper surface which increases the pressure difference across the wing and hence

${ }^{*} \mathrm{PhD}$ Candidate, Department of Aeronautics, Imperial College London, AIAA Member

†Senior Lecturer in Structures, Department of Aeronautics, Imperial College London, AIAA Senior Member.

¥Senior Lecturer in Aerodynamics, Department of Aeronautics, Imperial College London, AIAA Senior Member. 


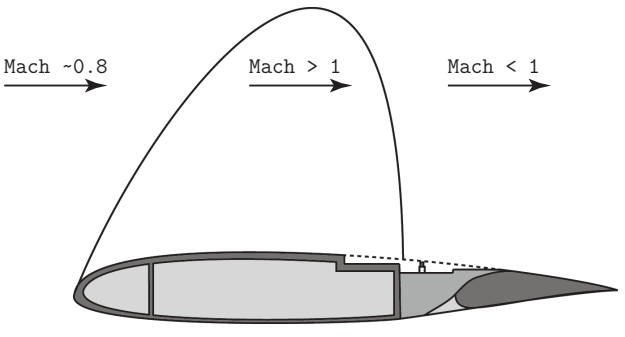

(a) Retracted SCB.

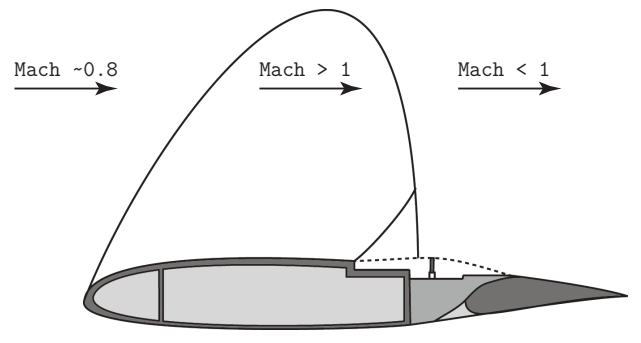

(b) Deployed SCB.

Figure 1. Typical global shock structure for transonic airfoils, sonic line shown.

the available lift. This makes transonic flight a potentially efficient flight regime where the extra lift may outweigh a negative effect due to the presence of a shockwave. To extend the favourable low pressure region, the size of the supersonic region is often increased; this is the case with natural laminar flow airfoils which involve a stronger shock which may need external control to avoid shock induced separation. Shock control bumps (SCB) are one such flow control concept and have been an established subject of research for many years ${ }^{2}$ and the focus of a recent review paper. ${ }^{3}$

$\mathrm{SCB}$ aim to reduce the wave drag of the airfoil by bifurcating the shock at the foot as sketched in figure 2 . This large scale shock structure reduces the entropy increase across the normal shockwave over a region close to the airfoil by introducing an oblique shock prior to the main normal shock. This gradual deceleration of the flow smears the large adverse pressure gradient.

A typical SCB consists of a ramp, crest and tail with a streamwise profile similar to that depicted in figure 2.4,5,6 The front shock leg is created by compression of the near-wall flow as it turns through the angle at the initial ramp incline. Front shock-leg strength is governed by the ramp angle, which is a function of bump length, crest location, and bump height. The crest is a smooth region, often flat, where the flow is

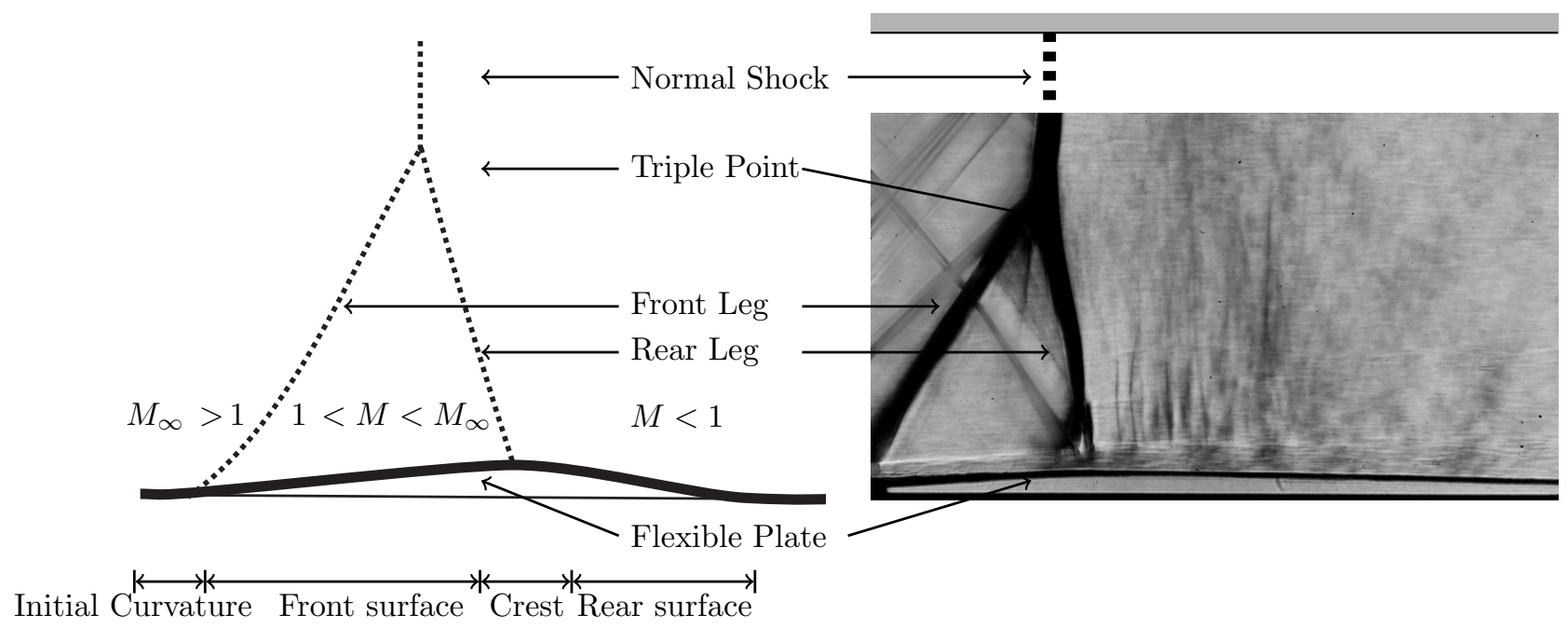

Figure 2. Definition of bifurcated shock terminology induced by flexible plate SCB.

gradually turned from the ramp on to the tail back towards the airfoil. The rear portion of SCB has recently become the focus of many studies ${ }^{7}$ which aim to control the flow over a much greater streamwise extent downstream of the SCB. The flow over the crest is very sensitive to surface curvature and re-acceleration of the flow over the crest of a SCB can occur if the control is placed in a non-optimal position. Effective SCB designs require very specific positioning with respect to the shock. If the bump is too far upstream then there will be significant re-expansion over the crest resulting in a strong shockwave at the rear leg. Positioning 
the SCB too far downstream of the shock will result in poor shock control and increase the likelihood of a secondary, local supersonic region over the crest. Both types of re-acceleration have a detrimental impact upon the pressure distribution which contribute to the poor off-design performance of current SCB.

It is the off-design performance of SCB that causes problems during the design phase. Throughout cruise the incoming Mach number will vary resulting in different shock positions. With traditional static SCB this would mean that an optimal SCB is incorrectly positioned for each shock location during an aircraft's cruise, leading to an undesirable shock structure and change in the wing $C_{p}$ distribution. As a result of this, the shock structure will change and a large scale pressure change will occur. One technique to avoid these off-design issues is an adaptive SCB that can deploy and retract. Such a device would be able to provide a performance benefit by always being on-design.

The design of an adaptive bump for shock control - the target of this work - has been discussed previously. ${ }^{8}$ The authors of this study claim that structure-integrated-actuation systems are required to enable an adaptive SCB to support the associated aerodynamic loads, and provide the required levels of deformation to generate a typical bump shape. They also proposed to integrate the adaptive device into wing spoilers to leave the principal aircraft design unchanged, and maintain the structural integrity of the wings. They introduced a shape parameterisation scheme linking the actuation and control requirements, which was subsequently optimized to find minimum actuation energy configurations. Unfortunately a lack of aerodynamic analysis means accurate estimates of wave-drag reduction were absent, however an estimated possible drag reduction of $14 \%$ was claimed.

An adaptive shock control bump of variable height is one way in which to achieve this morphing structure. The design and initial testing phases will be discussed through the analysis of on and off-design performance. This will highlight optimal design protocols for adaptive SCB and the integration to aircraft wings.

Morphing a SCB from an initially clean configuration offers the chance to produce a system which can perform optimally in multiple configurations. This paper demonstrates the feasibility of this approach via the generation of an optimization design framework targeted towards the design of adaptive structures in transonic flows.

\section{Adaptive SCB Design}

The design of a morphing SCB is a complex multidisciplinary problem, requiring both structural and aerodynamic analysis. ${ }^{9}$ This creates a large and nonlinear design space, with no analytical representation. Optimization is required to find a design that minimizes drag while exhibiting a sustainable level of deformation within the structure. In this study a gradient based optimizer explores the design space for SCB geometries through a weakly-coupled fluid-structure interaction solver. The central optimizer couples both a finite element structural analysis and a finite volume CFD solver. Each of these aspects will be discussed in greater detail in the following sections.

\section{A. Aero-Structural Optimization Framework}

In order to evaluate the performance of each design iteration a performance metric must be chosen. The lift and drag coefficients and their ratio, $C_{L}, C_{D}$ and L/D have often been used to quantify the performance of SCB however these global values tend to overlook the small scale effects which SCB are capable of affecting. The smaller scales are currently the subject of many experimental studies ${ }^{7}$ and should be included in the optimization. During a function evaluation, the aerodynamic properties of the actuated geometry are calculated through the use of CFD. The bifurcated shock structure in figure 2 reduces the total pressure loss in the region from the airfoil surface up to the triple point. Total pressure recovery downstream of the airfoil is a very good indicator of performance and has been used previously in studies of SCB, although predominantly for the analysis of specific SCB geometries rather than as a metric for optimization. Preliminary studies showed that whilst total pressure recovery downstream increased with the presence of a bump, the metric was not sensitive enough to differentiate between different bump designs. Therefore a performance metric that is more sensitive to the design variables is required. One possible option is a surface based performance metric which will take in to account the viscous effects around the SCB and includes the effects of small scale flow physics to the optimizer framework. 


\section{Definition of Performance Metric}

The goal of the surface based performance metric is to replicate the ideal structure through the pressure gradient over the SCB section. It aims to smear the pressure rise across the shock as much as possible and bridge the gap between optimizing for global variables $\left(C_{l}\right.$ and L/D) and small scale experimental studies. ${ }^{7}$ By focussing the performance metric in the region of control the values generated will be much more sensitive to changes rather than single $C_{l}$ values. The objective function is expressed mathematically below and schematically in figure 3 .

$$
\begin{array}{ll}
M=\left.\frac{d C_{p}}{d x}\right|_{\text {clean }}-\min \left\{\left|\frac{d C_{p}}{d(x / c)}\right|_{\frac{d p}{d x}=0 \mid}\right\} & \text { (pressure based performance metric) } \\
\text { s.t. } & \text { (stress contraint) } \\
\sigma_{\max } \leq \sigma_{y}(1-k) &
\end{array}
$$

For the results shown here, the safety factor $k$ is arbitrarily set at $10 \%$, and $\sigma_{y}$ is assigned the yield strength of the selected material.

The two stage pressure rise across the SCB reduces the chance of separation due to the adverse pressure gradient across the shockwave. Upon an airfoil the risk of separation is increased by both the initial curvature and the curvature added by the SCB. The performance metric and the optimization must be able to identify poor design choices and optimize efficiently.

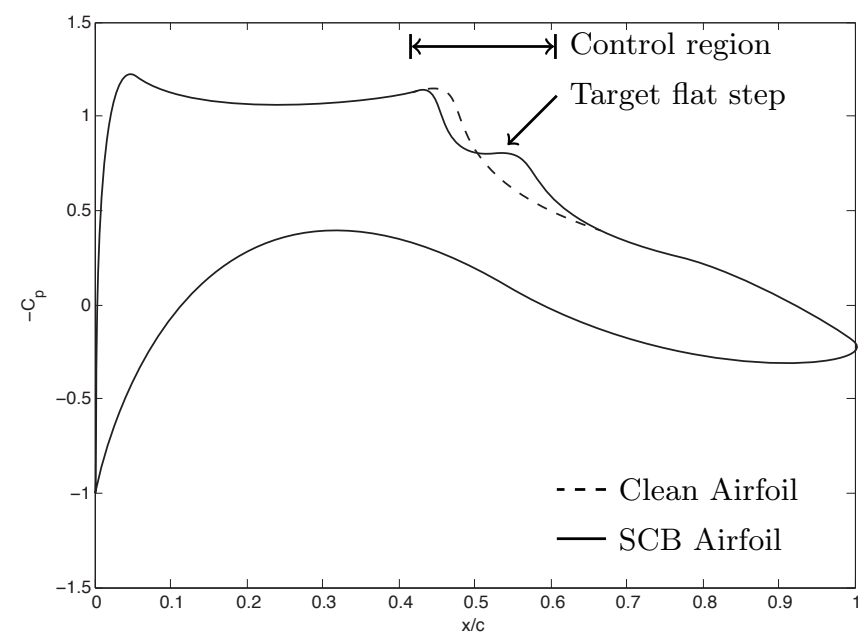

Figure 3. Schematic diagram illustrating target pressure coefficient distribution around airfoil.

The computation times are multiplied with optimization studies as multiple CFD analyses are required for each design variable. Therefore an efficient choice of design variables is required which are influential enough to affect the flow and sufficiently few in number to ensure quicker computation times.

The design variables are illustrated in figure 4 and were selected for the structural and aerodynamic influence. Bump height and ramp angle have previously been found to be key parameters. ${ }^{4}$ Overall height is represented in the design by actuation height, the ramp angle is a function of this height; due to the prescribed deformed plate geometry. A second actuation point is included in order to gain greater control over the crest region. Equation 1 shows the decision variables.

$$
\Omega=\left[\begin{array}{lllll}
B_{1} & x_{1} & \delta_{1} & x_{2} & \delta_{2}
\end{array}\right]
$$

$B_{1}$ dictates the start of the morphing region which is fixed at $0.2 \mathrm{c}$ (discussed in the following section), $x_{i}$ provides the chordwise location of the actuation on the pseudo-2D geometry and $\delta_{i}$ denotes the magnitude of the displacement applied. Nodes are created at these boundaries, and used to apply fully-fixed boundary conditions. Allowing the location to move with respect to the airfoil enables the best location of the SCB to be found during optimization. The structure outside these bounding nodes is fixed in order to remove any detrimental aerodynamic effects of an elastic response outside the morphing region. 
Small actuator displacements $\delta_{1}$ and $\delta_{2}$ are capable of producing a surface profile that has a significant beneficial impact on the flow at certain flow conditions whilst maintaining the key ability to retract and eliminate any off-design penalties. The optimization framework carries out the process outlined in figure 5. Each of the iterations is made up of a series of perturbations to the design variables in equation 1 in order to explore the design space. The optimizer uses the gradient based scheme fmincon in MATLAB which calculates an objective function via a series of perturbations to design variables. A centred finite-difference scheme is then used to calculate the optimal parameters for the next iteration.
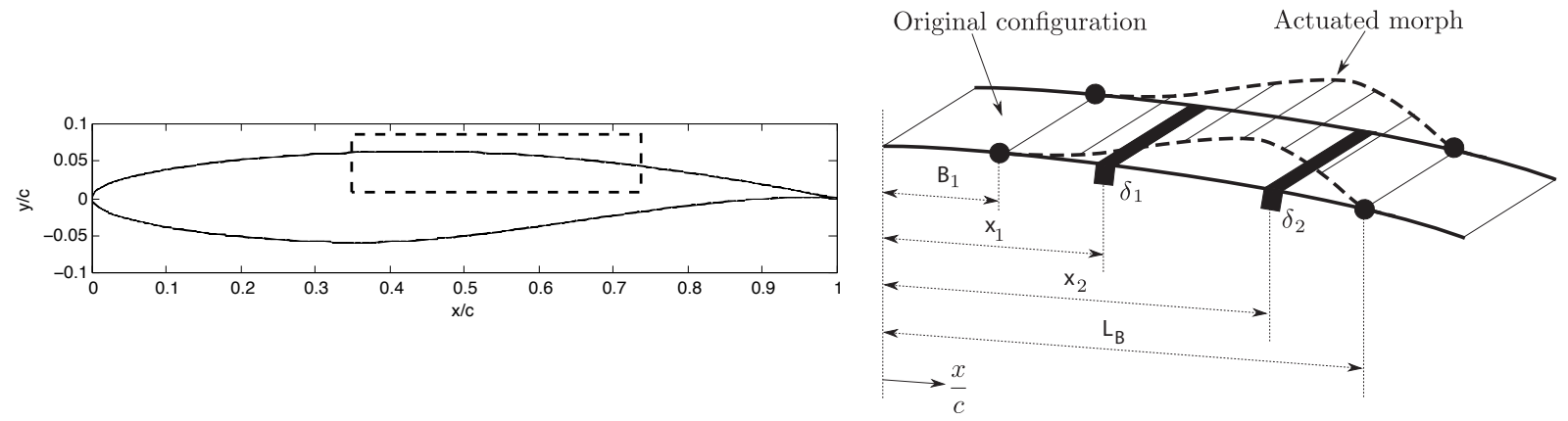

Figure 4. (a) The datum RAE2822 airfoil geometry. (b) Decision variables for structural morphing to be used during the optimization procedure.

The structural displacements ( $\delta_{1}$ and $\delta_{2}$, at $x_{1}$ and $x_{2}$ respectively) produce a deflected geometry. The coordinates of the upper surface are applied to the airfoil starting at $B_{1}$ in the range $0.35 c \leq x \leq 0.6 c$. The range of actuation positions is limited to $0.3 l_{b} \leq x_{1}, x_{2} \leq 0.8 l_{b}$ with $x_{1} \leq x_{2}$, these ranges were based upon previous studies ${ }^{10,5,11}$ and material constraints. Initially the bump height governed by $\delta_{1}$ and $\delta_{2}$ was unbounded to test the optimizer's ability to produce a successful SCB. Once the positive effect had been noted, bounds were put in place to explore the design space.

The structural model is loaded with the optimizer-defined actuation and the geometry is incorporated into the structured CFD mesh. The CFD is run until convergence, where the residuals $<10^{-6}$, at which point the pressure over the SCB region is extracted. This pressure field is then applied to the FEA model in conjunction with the actuated displacements. The defected surface geometry produced includes actuator and pressure loading and is used to create a new CFD mesh which is then run until convergence. Figure 5 details a single function evaluation, from input of decision variables, through to output of aerodynamic properties.

Due to the large computational expense of a single function evaluation, which is dominated by the aerodynamic analysis, a number of measures are taken to speed up the optimization process. Due to the structured grid generation, comparable numbers of cells are generated regardless of bump size or shape. Therefore to reduce solution time further, the flow is initialized to the developed condition for the solved test case of a clean airfoil. This reduces the computation time by approximately half, as fewer transient effects are experienced. Despite these efforts, function evaluation times are still relatively costly, taking an average of 6 hours on $162.6 \mathrm{GHz}$ Xeon processors with $32 \mathrm{~GB}$ memory to achieve aeroelastic convergence for each perturbation.

\section{B. Structural Modelling}

The start of the morphing region is limited to the aft half of the suction surface, $0.35 c \leq B_{1} \leq 0.6 c$, around the expected normal shock location $x_{\text {shock }}=0.5 c$. Here the shock position is defined as mid-value of the main pressure rise across the shockwave. It is assigned material properties of isotropic aerospace grade 7075 (T6 temper) Aluminum $(E=72 \mathrm{GPa}, \nu=0.33)$ and a thickness of $0.4 \mathrm{~mm}$ which allows for suitable displacements under the yield stress for actuation forces $\leq 100 \mathrm{~N}$. The decision variables for optimization shown in figure 4 , govern the magnitude and location of actuation applied to morph the structure. To respect the 2D modelling approach, all loading and boundary conditions remain constant in the spanwise direction.

The transition between aerodynamic and structural computations has required the clarification of aspect ratio of the plate and dimensions in order to simulate the structural response. Working with an airfoil of chord $1 \mathrm{~m}$ provides a bump length of $0.2 \mathrm{~m}$ which is equivalent to many static SCB currently being tested. ${ }^{12,13}$ This dimension allows for suitable wall-normal displacements of the plate within the elastic region. The fixed 


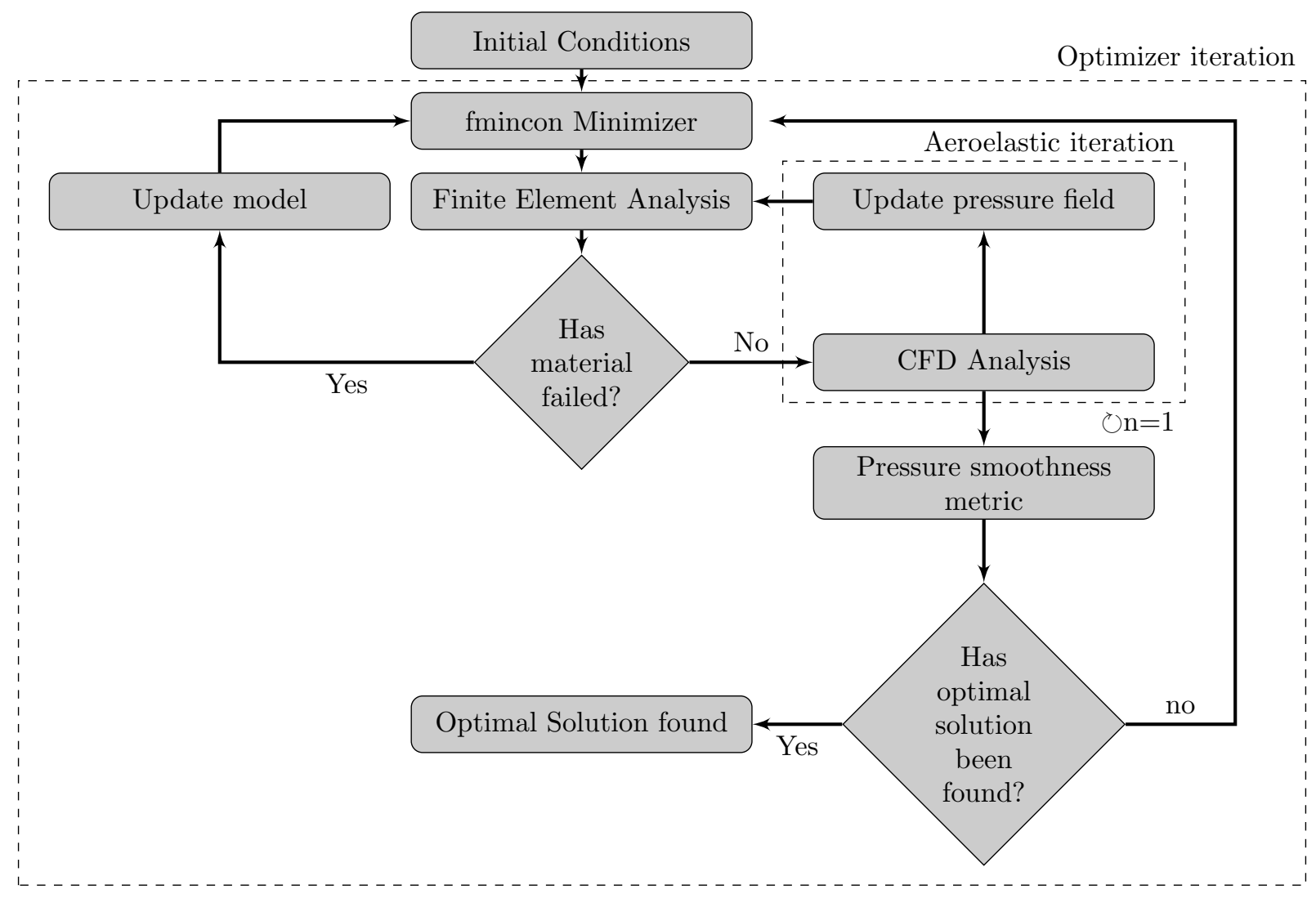

Figure 5. Flow chart for the optimization process. 
length also reduces the number of design variables and allows the flexibility of the plate to remain a function of actuation rather than include material effects.

These 2D geometries described by plate bending can replicate many of the existing shape families for typical SCB geometry. ${ }^{14}$ A plate subject to bending with just two control points can create a significant proportion of the polynomial and beam based geometries generated from non-structural and non-adaptive optimizations as well as reducing the number of variables required to describe the geometry.

The choice of beam-bending geometries has multiple benefits. First the surface maintains $C^{1}$ continuity; this ensures a smooth transition from the airfoil to the bump preserving flow quality. Second the mechanism required to deform the SCB with a continuous beam geometry is a much cleaner design as all of the actuation takes place out of the flow. This leaves the upper surface free of any obstructions and preserves the quality of the flow avoiding the generation of pressure waves or separation. With no hinge mechanisms connecting the airfoil and bump surfaces there is no possibility of leakage which could significantly affect the flow surrounding the SCB region. Lastly it enables the use of existing aerospace grade materials and proven actuator technologies in the design of adaptive SCB.

The commercial FE package Abaqus is used to compute structural displacements resulting from actuation. The structure is modelled using second order shell elements, and a geometrically nonlinear, quasi-static analysis is performed using a Newmark Algorithm with adaptive time stepping.

The decision variables have been set up in order to make suitable comparisons to existing literature regarding the flow physics. One of the aims of this paper is to introduce a local performance metric that quantifies the quality of the flow surrounding the bump region. Many previous experimental investigations ${ }^{7,15,12}$ were conducted in blowdown supersonic wind tunnels with working sections too small to instrument an entire airfoil. These geometric constraints resulted in SCB geometries which sit on the floor of the working sections with flat-base SCB. For this reason the optimization process adopted in this study considers SCB geometries with flat bases and maps the solution to the airfoil geometry.

In order to ensure that material deformations are elastic and the original retracted geometry can be regained after actuation it is necessary to limit the maximum deformation to a value below the yield point of the material. This limit will feature as a constraint in the optimization framework. To limit the maximum stress present during the morph, the von Mises equivalent stress is calculated over each element according to equation 2 .

$$
\sigma_{i, e q}=\sqrt{\sigma_{i, 1}^{2}-\sigma_{i, 1} \sigma_{i, 2}+\sigma_{i, 2}^{2}}
$$

$\sigma_{i, 1}$ and $\sigma_{i, 2}$ are the principal in-plane stresses for element $i$. The maximum equivalent stress forms the value to be constrained by the optimization.

$$
\sigma_{\text {max }}=\max _{i}\left\{\sigma_{\text {eqi }}\right\}
$$

The pressure field imposed on the plate by the fluid has a significant impact on plate deformation and this effect is considered in addition to stresses induced by actuation.

The stream wise pressure gradient associated with the wing's shockwave will cause a deformation of the thin aluminum plate. The pressure distribution over the flexible surface can be split in to two main regions. The first is the low pressure supersonic region between the front and rear legs of the main bifurcated shock. Second is downstream of the rear leg where the pressure is higher. In addition to these two regions above the flexible surface the pressure below the surface (cavity pressure) also plays an important role. The value used in this study for cavity pressure was selected following a preliminary investigation in to the effects of varied cavity pressure. Selecting a value below the post-rear leg pressure resulted in non-typical SCB geometries with depressions after the final actuation point. A higher pressure resulted in more traditional bump shapes although became more likely to yield.

The structural solver obtained the cavity pressure from the shock structure above plate was extracted from the aerodynamic analysis during each aeroelastic loop in figure 5. The dynamics of this interaction potentially move adaptive SCB towards more traditional panel flutter studies which require a time-resolved aero-structural solver. The quasi-steady solver described here evaluates the impact of the pressure loading on the structure and stems from traditional SCB design and optimization. 


\section{Aerodynamic Modelling}

In order to model the performance of SCB a suitable baseline airfoil is required. The modifications to the airfoil will specifically target the shock region so a typical supercritical wing with gentle upper surface curvature is selected. The RAE2822 airfoil provides a suitable baseline geometry with widely validated test cases. ${ }^{16}$ This feature of the airfoil lends itself to a larger variety of shock locations for subtle changes in pressure distribution than thicker airfoils.

The CFD analysis was completed using the rhoCentralFoam solver within OpenFoam. It solves the conservative form of density-based Reynolds Averaged Navier-Stokes equations through the finite volume method with Kurganov flux scheme coupled with the van Leer flux limiter method. The system is closed using the one-equation Spalart-Allmaras turbulence model due to the robust nature of the scheme.

\begin{tabular}{c|c|c|c|c} 
Re & $\mathrm{M}$ & $\alpha$ & No. Cells & Avg. $y^{+}$ \\
\hline $6.5 \times 10^{6}$ & 0.728 & 3.19 & $300 \mathrm{k}$ & 7.5
\end{tabular}

Table 1. CFD case information and mesh characteristics.

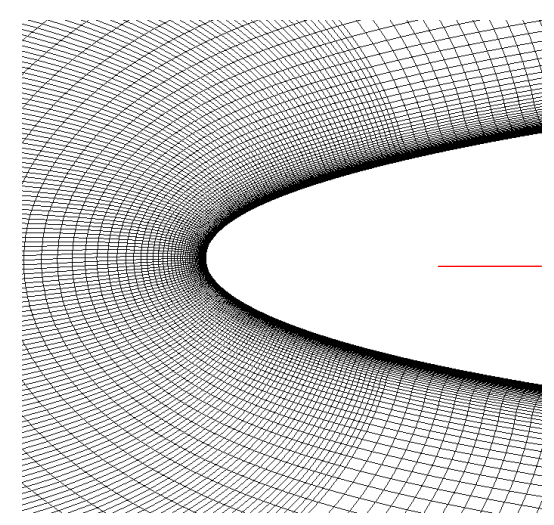

Figure 6. Mesh boundary and mesh refinement around the airfoil nose area (coarse grid shown for clarity).

Mesh refinements were added to the boundary layer and expected shock regions to better capture the flow, figure 6 , the first point was placed so that $y^{+} \approx 10$ in order to capture the physics in the boundary layer suitably close to the wall. The results have been compared to an experimental study on the same airfoil. ${ }^{16}$ This is shown in figure 7 and demonstrates good agreement between the model and the results from the experiment. This setup will be used in the optimization.

An additional concern is that in controlling the shock through a SCB, the lift distribution may be compromised, resulting in a variation of lift. In a real-world situation this would necessitate a change of angle of attack, altering shock position and potentially SCB effectiveness. It is therefore useful to monitor $C_{l}$ during optimization to ensure the required lift distribution is preserved during shock control. If the value is observed to vary by more than $1 \%$ the design is deemed ineffective and a penalty value is applied to the optimizer.

\section{Aeroelastic Design}

In order to facilitate a shape change that will affect the flow and without physically unachievable actuation forces, it is necessary to increase the flexibility of the structure. When considering a morphing system such as the wing skin in the current case, this means reducing the thickness and Young's modulus. However in reducing the stiffness, the system becomes more sensitive to aerodynamic pressure forces. Although the structural and aerodynamic aspects have until now been treated independently in optimization studies, ${ }^{5,8}$ it is necessary to investigate how they will interact in order to gain a complete understanding of how an adaptive SCB will behave in practice. The flow physics are heavily dependent upon the plate geometry with the large pressure gradients associated with shocks deforming the structure significantly. To determine the aeroelasticity of the problem, a weak coupling of the structural and aerodynamic solvers has been implemented. A quasi-steady analysis was performed initially to determine whether the system stays within 


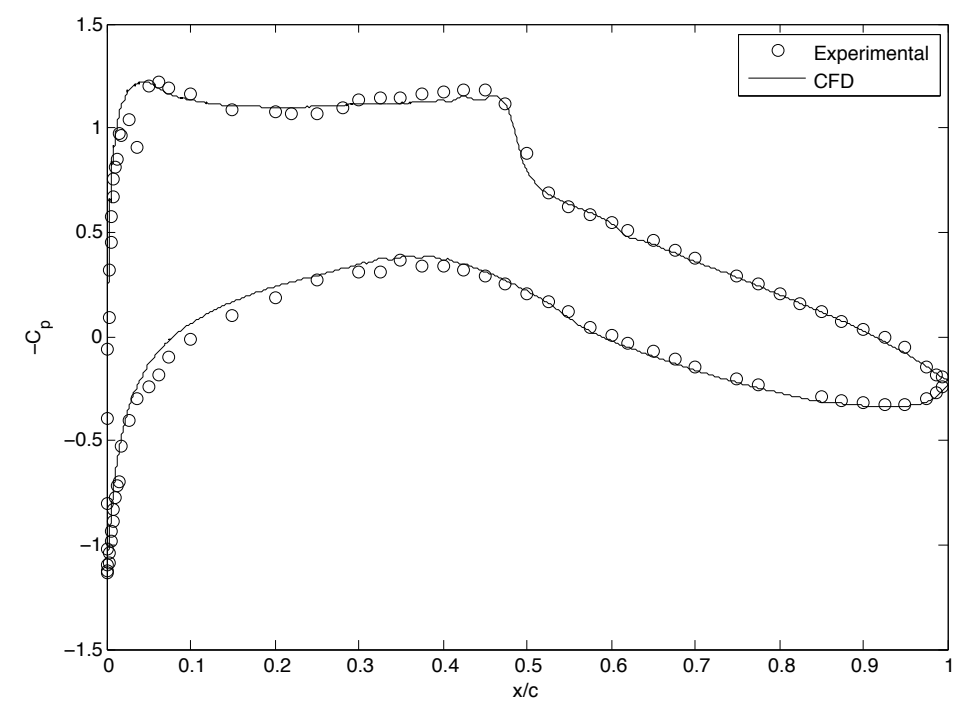

Figure 7. Experimental and computed CFD pressure profile comparison for the datum RAE2822 airfoil with no control measures, $\alpha=3.19^{\circ}, \mathrm{M}_{\infty}=0.728, \mathrm{Re}=6.5 \times 10^{6}$. Experimental data extracted from ${ }^{17}$

the elastic regime of the material when subjected to pressure loading. The aim of this optimization study is to include an element of the aerodynamic loading in the optimization metric.

As detailed in figure 5, the pressure field above the flexible plate is mapped from the CFD solution to the FEA model once. This is to include some of the effects of the pressure rise within the optimization. The coupling is limited to one iteration as it becomes very expensive for each perturbation. The decision to only complete one aeroelastic iteration of the aero loading was enforced once a preliminary quasi-steady study was completed. The coupled solver was run for 100 iterations and the global shock position, determined by mid-pressure rise, was found to oscillate to within 0.02c. This is within typical shock movement on transonic aircraft and an acceptable range for the quasi-steady analysis however to fully understand the coupling further time resolved investigation is required.

A study of transient effects during bump deployment is beyond the scope of this work, and would severely lengthen the optimization process due to the increased complexity and computational cost of a single function evaluation.

\section{Aero-Structurally Optimal SCB}

The aero-structural optimization procedure took on average eight iterations and 30 perturbations to produce an optimal design. This comprised of 15 initial perturbations to explore the design space via the centred finite difference scheme. Once the optimal design had been achieved a further two iterations were forced to check for local minima within the design space. The design space proved to be sensitive to the presence of a SCB, and the bump position had the greatest effect upon performance. The performance metric was not as sensitive to a change in height $\left(\delta_{1}, \delta_{2}\right)$ and even less so to actuation position along the $\operatorname{SCB}\left(x_{1}, x_{2}\right)$. The implications of the varying sensitivities upon on-design and off-design flight conditions are discussed in the following sections.

Figure 8 shows the variation of key parameters throughout the optimization process. The most influential decision variable was SCB position as it had to be located specifically to match the global shock location determind by the pressure field surrounding the airfoil. The optimizer recognises the dominance of SCB location and the evolution of optimal SCB placement can be seen in figure 8d. Table 2 shows the both initial conditions of the optimizer and the subsequent optimal solution.

The effects on the overall lift are minimal, with the off-design changes in pressure distribution shown in figure 12 resulting in a maximum change of $0.5 \% C_{l}$ which is shown in figure $8 \mathrm{~b}$.

The freestream supersonic region and shock location remained relatively stable throughout the iterations with significant changes only occurring within the shock-foot region. Most notable was the shift from the weak shock-boundary layer interaction (SBLI) to a lambda shock. This is visible at $\mathrm{x} \sim 0.5 c$ between the 

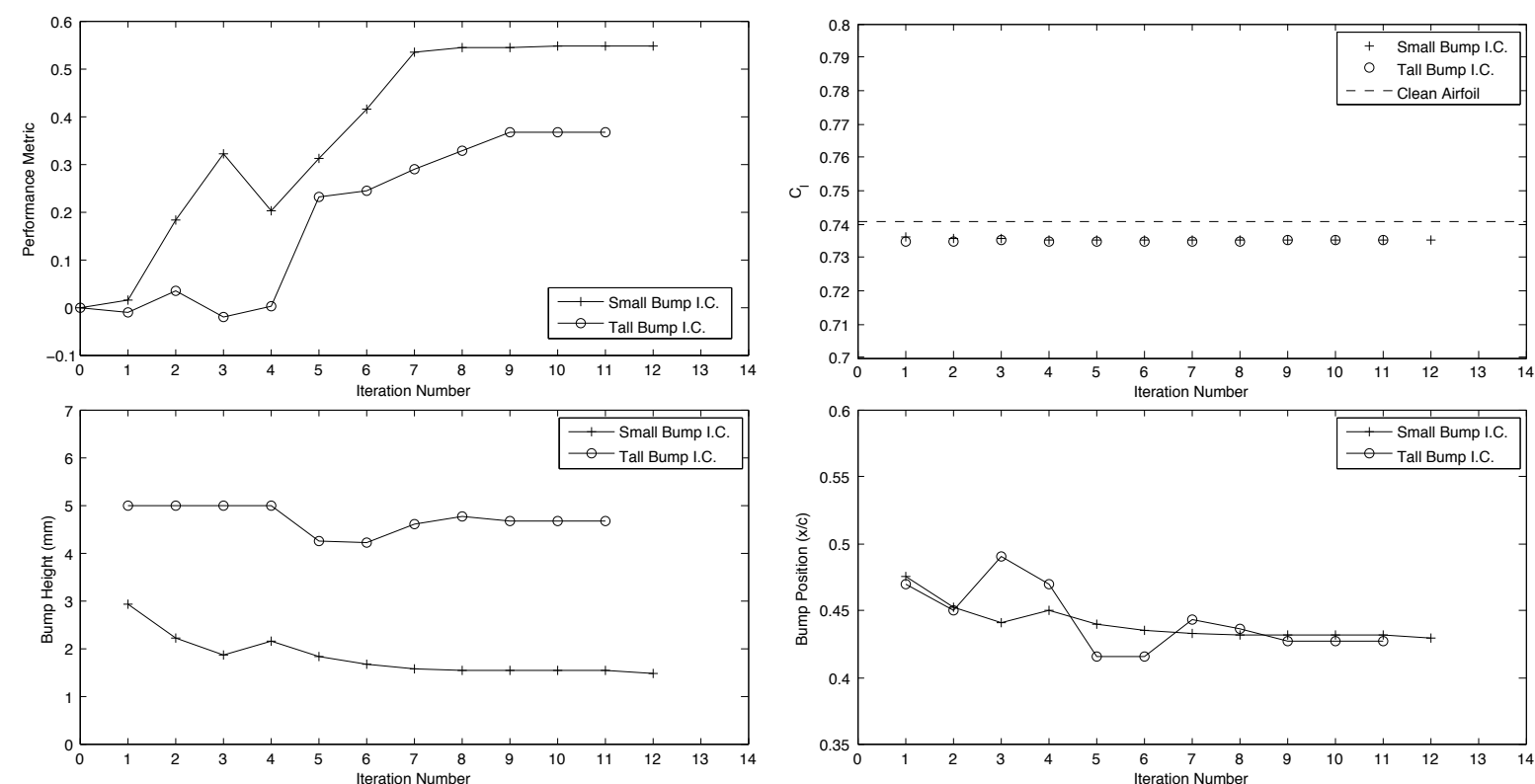

Figure 8. Key performance data through the iterations of the optimization procedure. Clockwise from top left: Performance metric, lift coefficient, bump position and bump height. Where presented, iteration 0 marks the clean airfoil.

\begin{tabular}{l|c|c|c|c|c|c|c} 
& $B_{1}(\% C)$ & $x_{1}\left(\% l_{b}\right)$ & $\delta_{1}(\mathrm{~mm})$ & $x_{2}\left(\%_{b}\right)$ & $\delta_{2}(\mathrm{~mm})$ & $\sigma_{\max }(\mathrm{MPa})$ & $\mathrm{M}$ \\
\hline Initial Condition & 0.47 & 0.5 & 0.3 & 0.55 & 0.3 & 170.02 & 0.57 \\
\hline Optimal Solution & 0.43 & 0.55 & 1.49 & 0.57 & 1.49 & 308.4 & 0.5491
\end{tabular}

Table 2. Optimal SCB decision variables for the initial condition and optimal solution, 2 point actuation. 
experimental result and the region labelled SCB in figure 9.

The RAE2822 airfoil has a natural shock position at $x=0.495 c$ for $\mathrm{M}=0.728 \alpha=3.19$ this is situated close to the point of maximum thickness at $0.5 \mathrm{c}$ allowing for a relatively stable shock position. In order to design an effective $\mathrm{SCB}$, the ramp section must give a pronounced departure from the existing airfoil surface. Placing the SCB too far upstream prior to the $y_{u, \max }$ location will continue to accelerate the flow to higher Mach numbers (compared to design) without imparting the lambda structure. This is illustrated in figure 10a with the supersonic region extending beyond the crest and increasing the strength of the shock. Additional viscous losses were also incurred as the stronger shock caused boundary layer separation. Figure 10c shows the SCB positioned too far downstream which causes significant reacceleration of the postshock flow potentially increasing boundary layer thickness. The resultant expansion waves caused large scale curvature of the rear leg weakening it slightly however closer to the wall the effects of the curvature and the acceleration resulted in a stronger shockwave. For an optimal solution, figure 10b, a bifurcated shock structure has been achieved without any regions of reacceleration. The optimizer manages the curvature of the plate region through small changes in the decision variables which can be seen to affect the flow structure significantly.

The curvature of the rear surface of the SCB is determined by the combination of actuation and the pressure loading. The FEA results in figure 11 show the geometries and stress distribution surrounding the optimal plate due to the actuation. The addition of the pressure load to the optimal design significantly increases stresses, particularly at the constraints. The largest stress is a combined result of the pressure and displacement loading at $x=0.43 c$ and places a material limit on the designs of adaptive SCB.

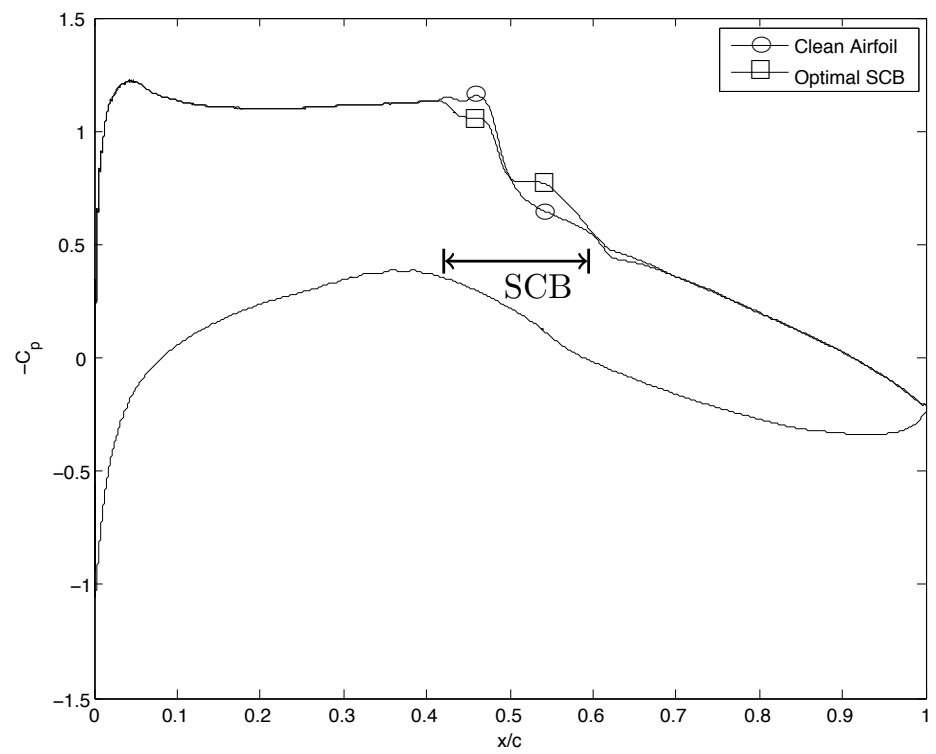

a)

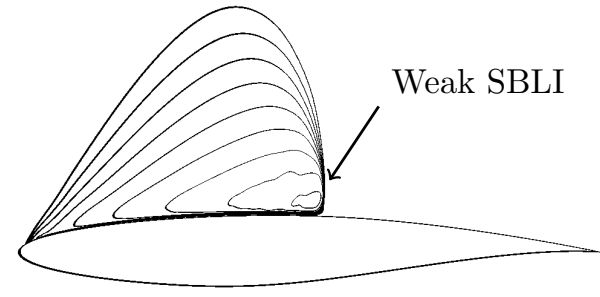

b)

Figure 9. The effect of introducing a SCB. a) Pressure coefficient profiles for the clean airfoil, $\alpha=3^{\circ} 19^{\circ}$, $M_{\infty}=0.728, R e=6.5 \times 10^{6}$. b) Mach contours for the clean airfoil.

Figures $8 \mathrm{a}$ and $8 \mathrm{c}$ show that as actuation height becomes smaller the optimizer progresses towards an optimal solution. Structurally, the plate exhibits a maximum stress that is just $59 \%$ of the yield stress of the material which provides a very large safety factor in terms of material failure by plastic deformation and removes the concern that these plates will fail due to the aerodynamic pressure loading.

SCB performance over a range of bump heights is an important question for adaptive SCB in order to predict the aerodynamic performance during bump deployment. To investigate this, a parametric study exploring the design space has been carried out and the results are reported in the following section. 


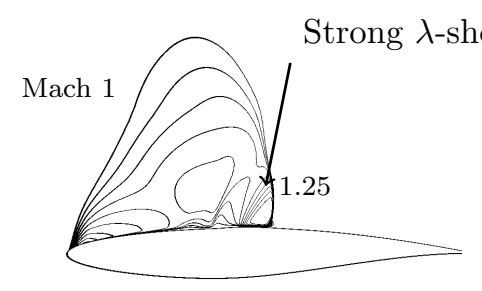

a)

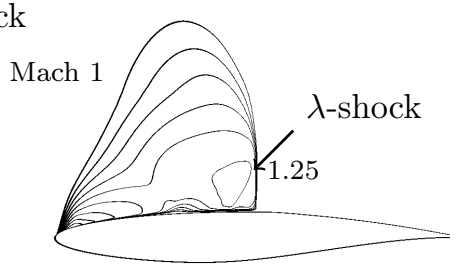

b)

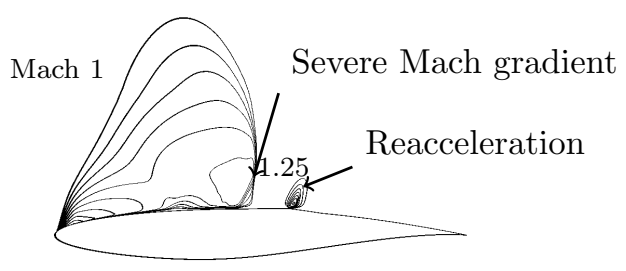

c)

Figure 10. Mach contours showing the supersonic regions for a) upstream $\mathrm{SCB}\left(\mathrm{B}_{1}=0.36, \mathrm{x}_{1}=0.55, \delta_{1}=3\right)$. b) optimal solution, $\left(\mathrm{B}_{1}=0.43, \mathrm{x}_{1}=0.55, \delta_{1}=1.49, \mathrm{x}_{2}=0.57, \delta_{2}=1.5\right)$ and $\left.\mathrm{c}\right)$ downstream $\mathrm{SCB}\left(\mathrm{B}_{1}=0.43\right.$, $\left.\mathrm{x}_{1}=0.55, \delta_{1}=3, \mathrm{x}_{2}=0.57, \delta_{2}=2.4\right)$
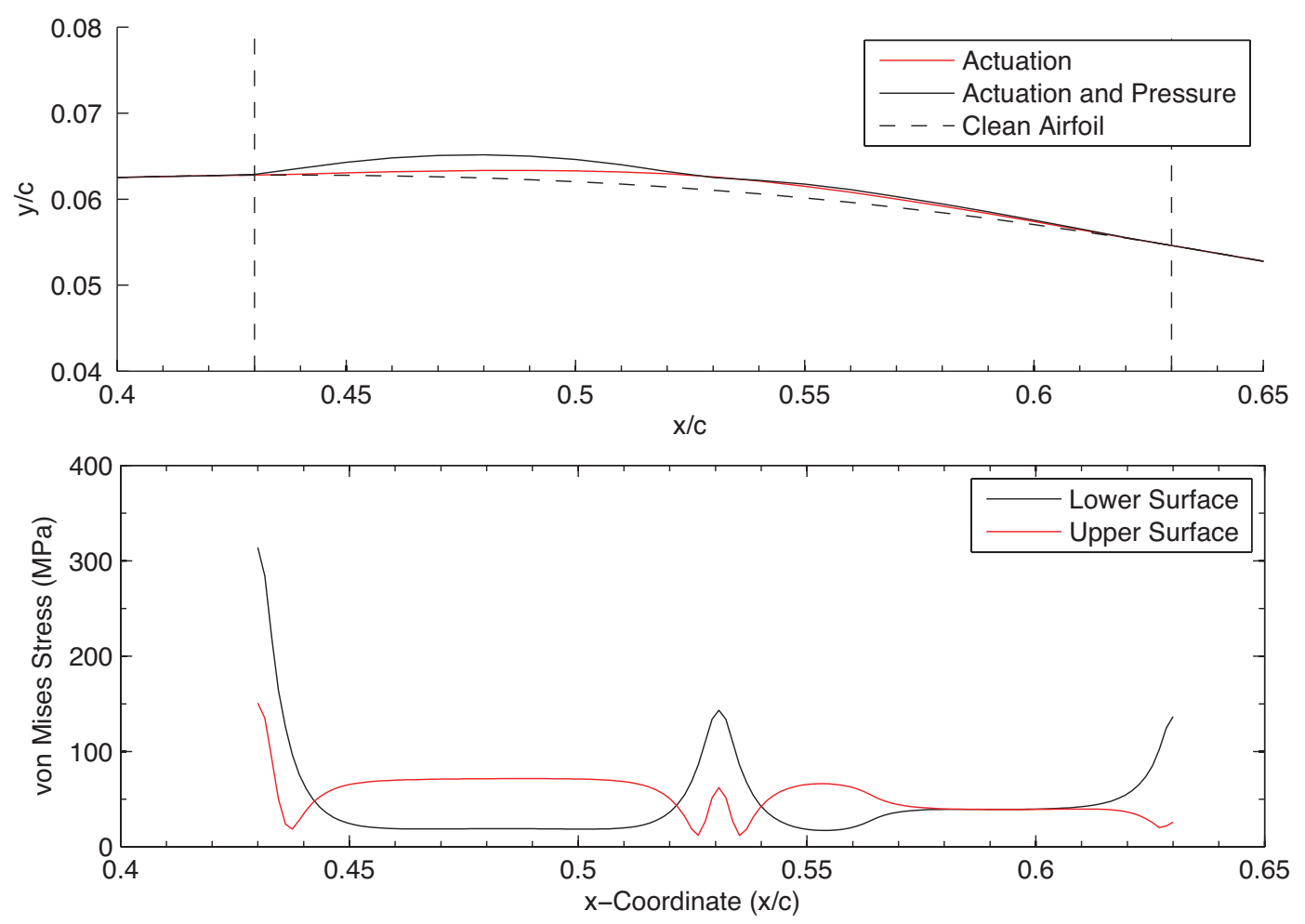

Figure 11. Structural modelling of adaptive SCB. Surface and centreline vertical displacement and centreline von Mises Stress for upper and lower surfaces. 


\section{Off-design and Aeroelastic Performance}

During cruise, aircraft are often required to vary speed and altitude. These manoeuvres could change shock location with respect to any SCB installed on a wing. ${ }^{15}$ Throughout the optimization process numerous perturbations are applied to the initial design variables to find an optimal value. These perturbations can have positive or negative effects as the optimizer explores the design space. In this section we explore the so-called off-design performance of a wing with a SCB and how the deployment of a morphing SCB may impact performance.

\section{A. Off-design Performance}

Non-optimal solutions encountered during the optimization process provides a wealth of information about off-design performance. Pressure fields from all of the perturbations have been analysed to investigate the effects of a non-optimal design. The most influential variable in overall bump performance is SCB location, in particular the position of the shock relative to the crest of the SCB. This dependency has been identified previously as an important design variable. ${ }^{6}$ The exact location of the shock is difficult to extract from surface pressure measurements due to viscous smearing. The second most effective design variable is the bump height; this dictates the flow quality over the SCB and the overall performance for a given SCB location.

Grouping the results of both the parametric study and the optimization perturbations with respect to height and position provide a detailed view of the design space. Figure 12 shows how the actuation height of the SCB crest has a significant effect when it comes to maximising performance. This value represents the pressure gradient in the bump region and represents how smeared the pressure rise is across the shock.

The SCB in figure 12 show the performance variation with bump height for non-yielding solutions. The limits imposed by the structural constraint were in place during all the perturbations and restricted the overall height and shape of the SCB. For all locations, the presence of a tall bump caused a significant drop in performance. The undesirable shock structures in figures 10a and 10c are often encountered with taller SCB.

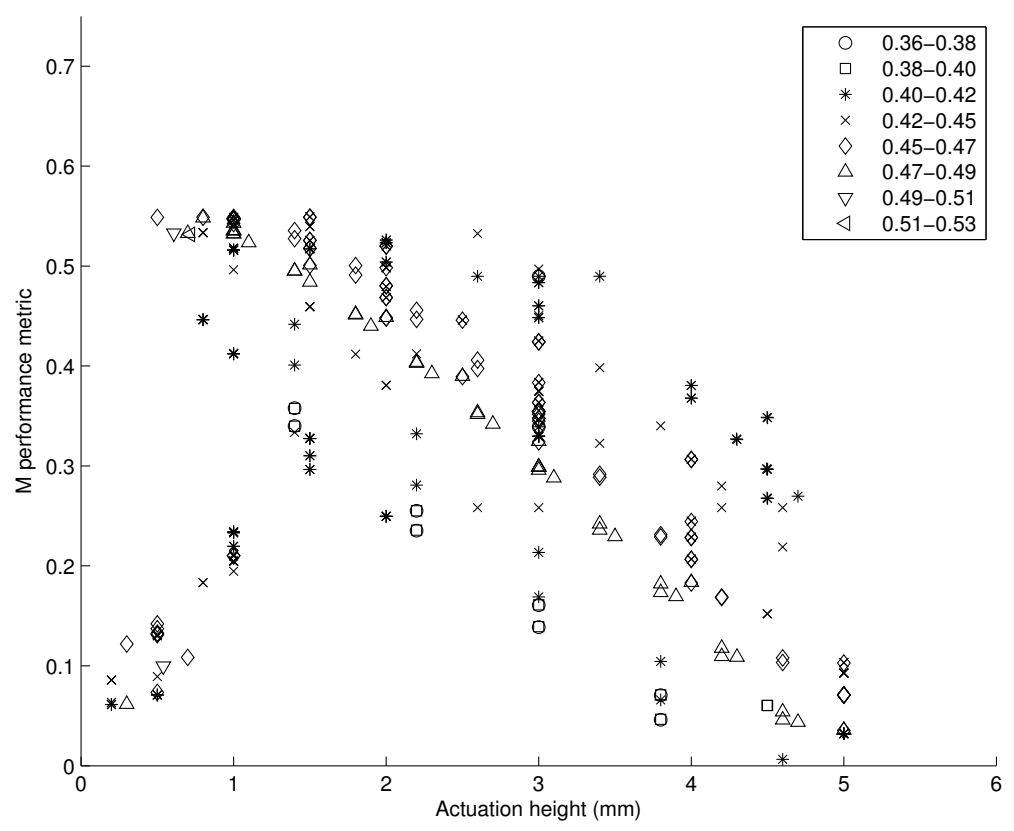

Figure 12. Performance vs. bump height grouped by stream wise positions. The data was extracted from multiple optimization and parametric runs.

The main trend in figure 12 is that taller SCB have worse performance than shorter SCB with the 


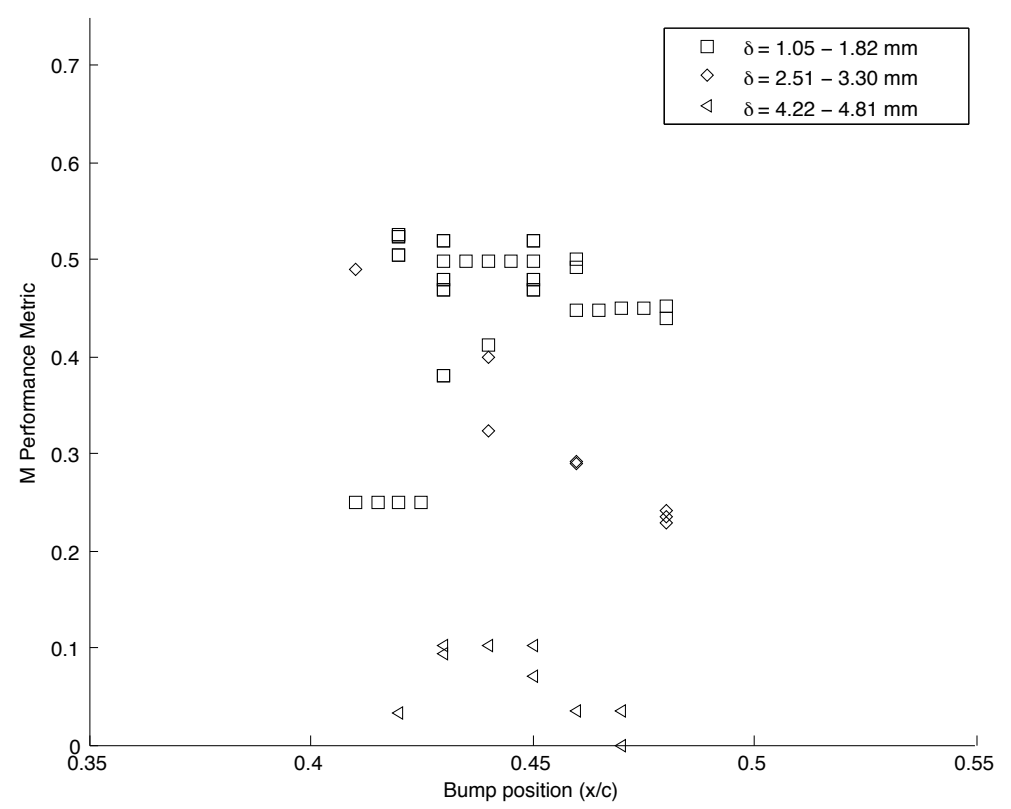

Figure 13. Performance vs. bump position grouped at various heights. Reduced dataset compared to figure 12 for clarity

performance metric dropping from around 0.55 to 0 as the actuation heights $\left(\delta_{1}, \delta_{2}\right)$ are increased. The exceptions to this are for small actuation heights (typically $\delta<1 \mathrm{~mm}$ ) where performance is poor.

The reason for these two distinct groupings is due to the shock structure. As a SCB is deployed there is some positive effect as the pressure rise is smeared and the pressure gradient is reduced sightly resulting in increased performance. It is at a displacement of $0.5 \mathrm{~mm}$ at which the bifurcated shock structure is first achieved (upper left quadrant). It is this snap between the two structures that forms the lower bound of pressure loaded SCB heights. Exploring this lower bound of bump actuation heights is important as the detrimental viscous effects seen with higher bumps are minimised.

The position of this first $\lambda$-shock inducing bump $\left(B_{1}=0.45 c\right)$ places the shockwave at $0.25 l_{b}$ which is just on the front surface of the SCB. This placement is very sympathetic to the flow as there is still a significant stream wise region of small curvature after the shock before the flow is subjected to the large convex curvature around the crest which minimizes the likelihood of curvature induced separation.

By identifying the groups of bump position in figure 12 it can be seen that there are some better performing locations. The common trait is that the best performing designs place the crest immediately downstream of the shockwave. The effects of separation are discussed further in the following section but these optimal designs balance the ability to initiate an efficient bifurcated shock and deal with the surface curvature.

Figure 13 shows the performance variation with bump start position. Alternate height ranges were selected to show banding between SCB heights. The performance jump identified in figure 12 between a single and bifurcated shock structure can be seen for the smallest actuation heights, $1.05<\delta<1.82 \mathrm{~mm}$. The drop in performance can be seen as the position of the SCB progresses downstream. Slightly taller SCB $2.51<\delta<3.30 \mathrm{~mm}$ show how relatively good performance can be achieved when the SCB is optimally positioned $B_{1}=0.43 c$. As the SCB is positioned too far downstream the performance drops off much quicker than the smaller SCB. This goes someway to highlight the wider performance envelope of smaller pressure loaded SCB as they are more effective for a wider range of shock positions with fewer off-design flaws.

The sensitivity of the flow to the shock position/crest location ratio has been previously documented ${ }^{6}$ and has been confirmed here. The shock position on the clean airfoil was calculated to be at $0.495 \mathrm{c}$. With crests found near $0.5 l_{b}$ the best performing bumps were within the range $0.43 \leq B_{1} \leq 0.45 c$. Within this range the SCB actuation location is between $0.51 \leq x_{1} \leq 0.575 \mathrm{c}$ and places the rear shock leg on the front face of the SCB. This is the traditional position governed by the overall pressure field with subsonic flow down the rear face of the SCB. The structural constraint seems to have little effect on global shock position 
for a given flow condition.

The position of the crest with respect to the start of the bump, $x_{1}, x_{2}$, had little effect on the performance metric with no correlation found. The range covered by the optimization was $0.3 \leq x_{1}, x_{2} \leq 0.7 c$ which was primarily restricted by the structural constraint. A SCB with large deflections positioned outside this range would produce very large stresses. The optimizer would then apply a penalty value, to avoid running expensive CFD analysis, bounds are introduced to guide the exploration of the design space efficiently.

Requiring smaller bumps limits the stresses within the system and would prolong the life of the system with respect to fatigue loading. Repeatable deployment is one of the key of adaptive SCB and this optimization study has highlighted that flexible plates do remain within the elastic regime.

From figure 12 and 13 the best performing bump heights are within the range $0.8 \leq \delta_{1} \leq 1.7 \mathrm{~mm}$ and the addition of a structural constraint to the optimization has not limited the bump heights nor their ability smear the pressure rise. Figure 14 shows the effect of varying SCB height and how the pressure distribution changes. These profiles represent bumps at the optimal position, $B_{1}=0.43 c$. The airfoils with larger actuation displacements suffer from large low pressure regions between $0.52 \leq x \leq 0.63 c$ which include regions of separated flow. This is because the curvature of the plate around the crest region is too great for the flow to remain attached.

As the actuation height increases, the maximum ramp angle of the front surface of the SCB increases, together with the strength of the oblique shock. This can be seen by the increasingly sharp rise in $C_{p}$ observed at the very upstream extent of the control region, $0.41<x<0.43 c$. A greater initial shock strength reduces the Mach number present over the front surface of the SCB and reduces the strength of the rear leg of the $\lambda$-shock as illustrated by the smaller rise in $C_{p}$ around $0.47<x<0.50 c$. This minimises the chance of post-shock separation due to the adverse pressure gradient.

Figure 15 shows how the stream wise wall shear stress varies along an optimally positioned SCB with the same range of heights. For solutions shorter than the optimal the flow does remain attached over the SCB. For taller SCB $(\delta>1.75 \mathrm{~mm})$ a separation bubble appears around the crest. For very large actuation heights $(\delta=4 \mathrm{~mm})$, an additional separation bubble appears at the start of the SCB ramp.

\section{Global Aerodynamic Peroformance}

The aero-structurally optimal SCB do not cause the flow to separate over the crest which shows the effectiveness of the performance metric. In contrast to other optimization studies the performance metric used here was designed to include an element to measure the quality of the glow in the control region at the optimiser level. To compare with previous optimization studies ${ }^{6,13}$ the global variables such as $C_{l}, L / D$ are analysed to assess the SCB performance. Figure 16 shows the $L / D$ values for an optimally positioned SCB for the range of heights also shown in figures 14 and 15.

All SCB airfoils show a slight improvement in the $L / D$ value with the largest improvement corresponding to the greatest actuator displacement tested at this location. The maximum improvement in $L / D$ was quite small with $\Delta L / D<0.6 \%$ with respect to the clean airfoil. By analysing the pressure profiles from figure 14 and the resultant lift-drag components the source of the $L / D$ was found to be a slight increase in $C_{l}$ as actuation height increased. The overall $C_{l}$ value for all bump heights was found to be $<0.5 \%$ lower than the clean airfoil case. This was due to the large low-pressure region that developed downstream of the crest for taller SCB increasing the lift. There were minimal changes in $C_{D}$ however these would be amplified at higher cruise Mach numbers. If the performance metric were to focus upon $C_{l}, L / D$ then the taller SCB would have proved optimal however these tall SCB perform poorly when the viscous effects are included as in this study. The choice of performance metric is therefore very important when it comes to optimising $\mathrm{SCB}$ geometries for various roles whether shock control or global $C_{l}, C_{D}$ performance.

\section{B. Optimal SCB Aeroelastic Performance}

The construction of the optimizer incorporates an element of the aeroelastic response by recalculating the FEA model with the aerodynamic load applied. The results generated by this process are shown in table 2 To assess the aeroelastic performance of the optimal SCB the actuated plate cases are subjected to repeated pressure loading. As the aeroelastic solver advances through the iterations the pressure loading increases surface deflection beyond that controlled by the actuation. Due to the pressure gradient across the plate the actuation points effectively became restraints limiting plate movement into the free stream. Figure 17 

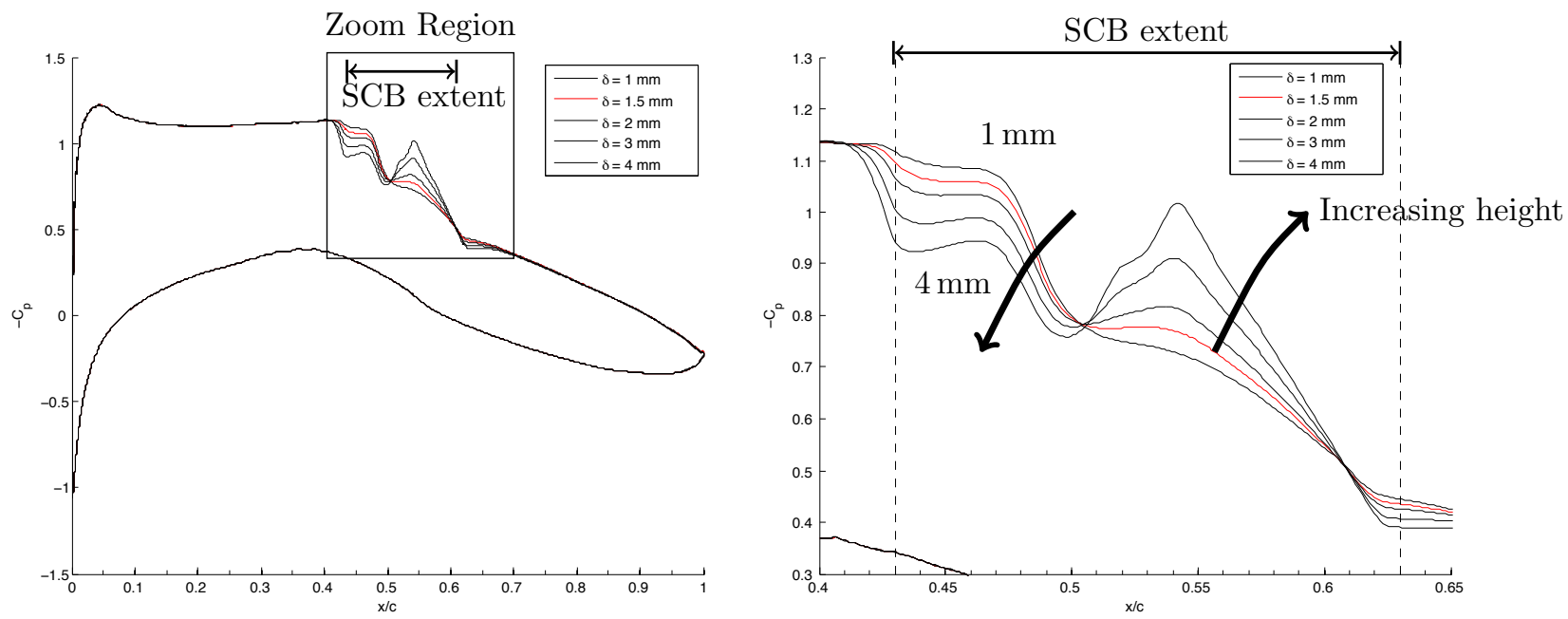

Figure 14. Pressure coefficient profiles for optimally positioned SCB with varying bump heights.

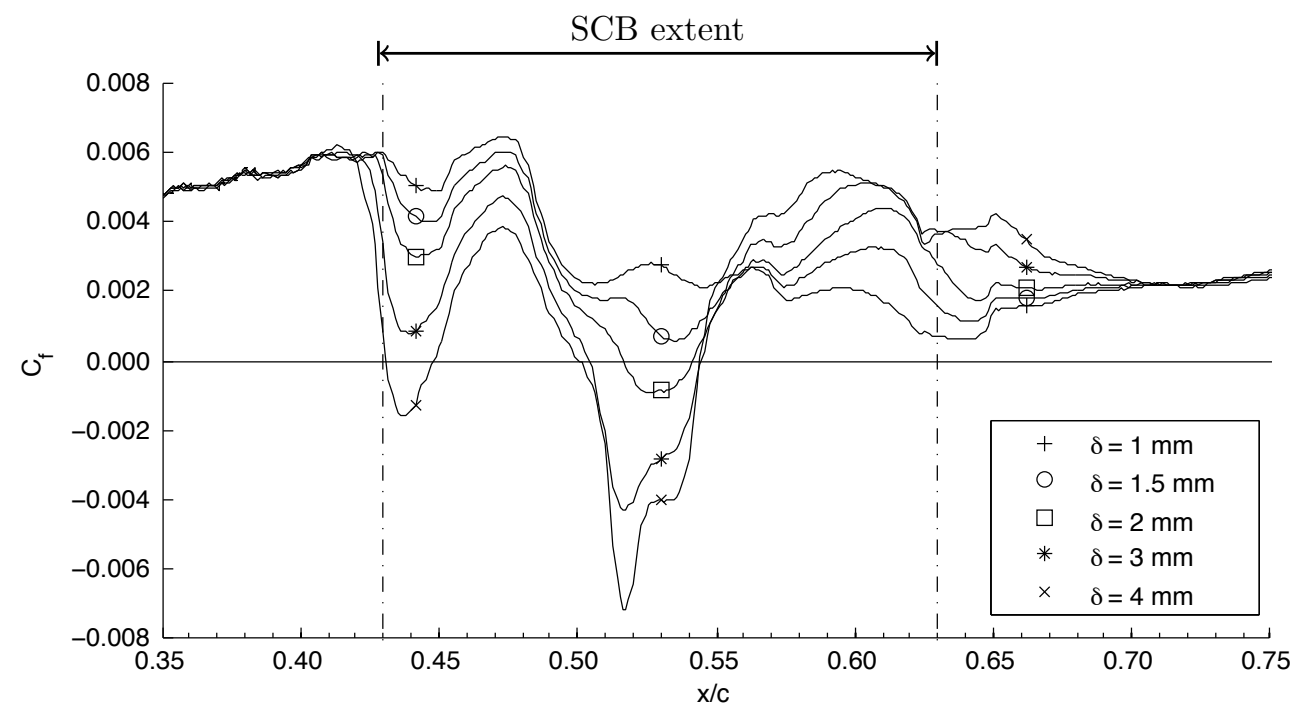

Figure 15. Wall shear stress coefficient for varying bump height in the optimal location $(\mathrm{x} / \mathrm{c}=0.43)$. 


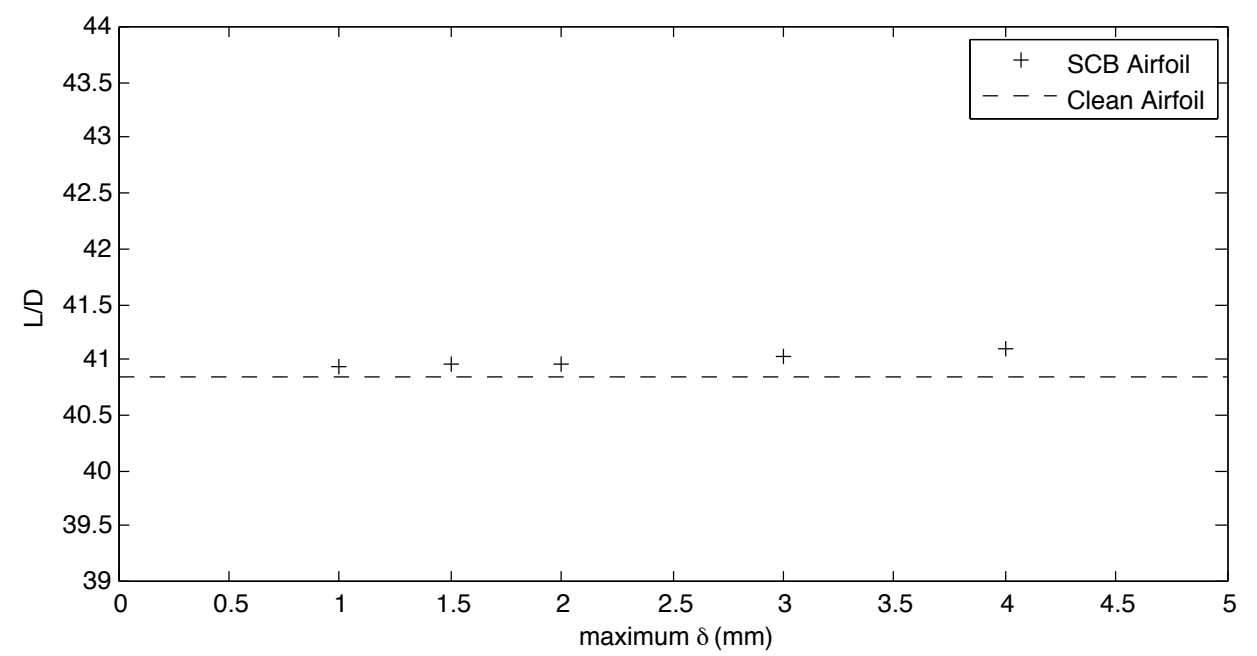

Figure 16. L/D variation for increasing bump height. Clean airfoil L/D ratio included for comparison.

highlights the difference between the geometries due to actuation alone and the combination of actuation with pressure loading.

Initial tests upon the aeroelastic response of the flow-plate interaction were carried out using the quasisteady aero-structural solver described previously. It is shown that for a flexible plate with no actuation and shock pressure loading that the response of the plate remained stable, albeit with poor performance. Over the test period of 100 iterations the shock structure remained almost stationary $\left(x_{\text {shock }}=0.53 \pm 0.02 c\right)$ with little $C_{l}$ variation $\left(\Delta C_{l}<0.5 \%\right)$. The stresses within the material remained within the elastic regime and so returned to a flat surface once the aero-load had been removed.

The incorporation of the optimal actuation positions and heights is shown in figure 17 . The low pressure supersonic region between the two shock legs causes the front surface to rise up from the original position. This new peak $y / c$ position is located at $0.48 \mathrm{c}$ whereas with solely actuation, the peak $y / c$ value was $0.55 \mathrm{c}$. As the structural solution is mapped to the geometry it can be seen how the actuation positions $\left(x_{1}, x_{2}\right)$ vary depending upon the curvature of the airfoil at that particular region. The pressure loading shifts the peak upstream from the point of actuation.

As the pressure loading continues to be applied, the low pressure over the front surface of the SCB causes further deformation. The increased plate displacement increases the maximum ramp angle at the front of the plate to $3.6^{\circ}$. This value is the peak angle and shows the benefit of plate-bending geometries which gradually increase and decrease the curvature causing further pressure smearing. For the optimal solution, actuation alone only produces a maximum turning angle of $0.5^{\circ}$. The effect of pressure loading is large and must be taken into account to a greater extent in the optimization, this means more aeroelastic loops must take place within each optimization.

These gradual deflections help introduce the desired oblique shockwave whilst maintaining relatively low level of curvature. The link between flow curvature and surface curvature has been identified previously ${ }^{7}$ where the shock position was changed to force off-design conditions. In their study the shock position was moved from the crest at $0.5 l_{b}$ to $0.63 l_{b}$. These two shock positions have highlighted that curvature is of utmost importance in the $\lambda$-shock region as well as the SCB crest and shock location ratio.

In the present study, the effects of plate curvature can be seen in the wall shear stress distributions in figures 18. The geometries with higher displacements have higher curvatures around the crest. This will cause the flow to either separate or re-accelerate and strengthen the downstream shock. Such a shock structure can be seen in figure 19. There is some reacceleration over the crest however the flow remains attached due to the reduced Mach number. The crest Mach number is dictated by the strength of the oblique shock which is governed by the curvature at the front of the SCB region. It can be seen that a trade-off is required between maximising the curvature at the front and minimising over the crest.

The geometry exhibited by the plate after pressure loading remains comparatively unchanged over the rear section downstream of any actuation points, figure $17,0.52 c<x<0.63 c$. The significant difference observed in the upstream region highlights the effect of low static pressure in the bifurcated shock region. 
It also suggests that where the pressure gradient across the plate is large there is a need for more actuation points to limit the pressure induced displacement.

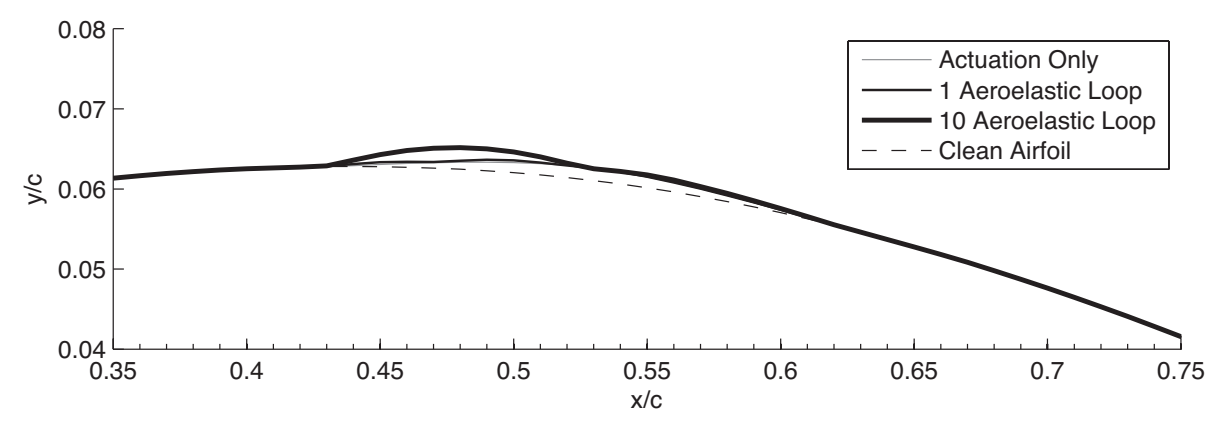

Figure 17. The effects of pressure loading upon actuated SCB, actuated SCB subject to a further 9 iterations of the aeroelastic calculation and that of a clean airfoil.

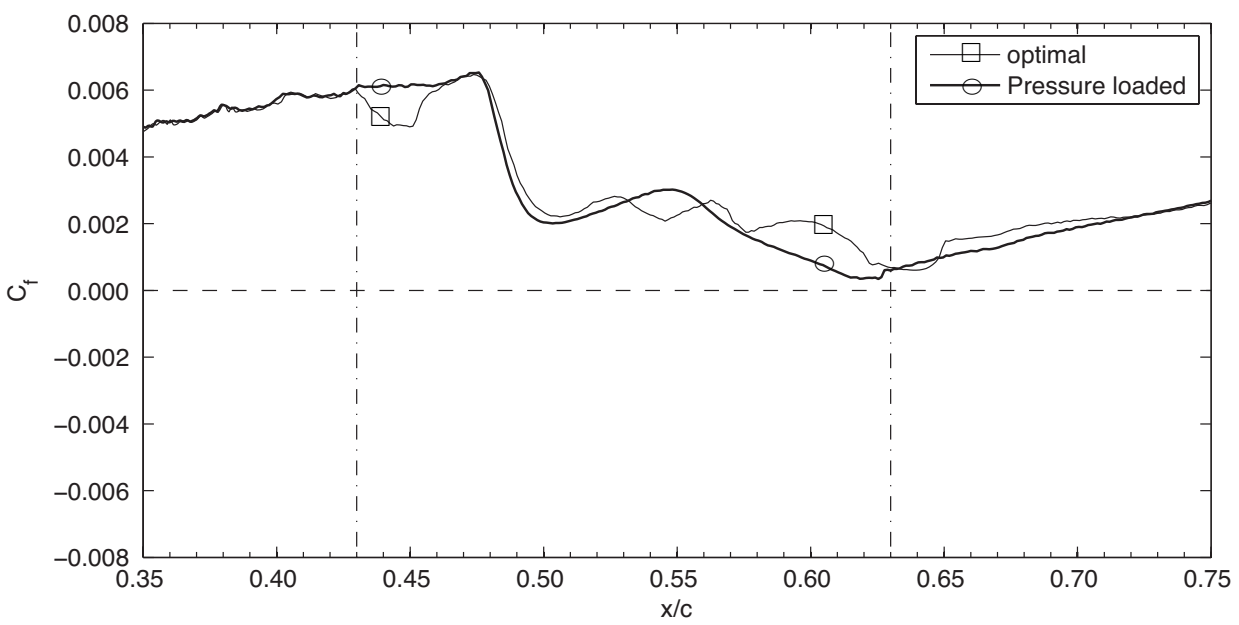

Figure 18. Wall shear stress profiles showing the effects of continued pressure loading to the adaptive SCB.

\section{Conclusions}

The concept of adaptive SCB has been investigated from the perspective of an optimization study. In particular whether an actuator based design using existing aerospace grade materials can perform in the vicinity of the shockwave. The flexibility requirements to achieve suitable bump geometries counter the robust design necessary to withstand the loads. This optimization framework has included a structural element that enables the material constraints to feature within a traditionally aerodynamic problem. It was found that the structural constraint does not restrict the range of designs evaluated by the optimization framework with maximum von Mises stress peaking at $308 \mathrm{MPa}\left(58 \% \sigma_{y}\right)$ for the aero-structurally optimal design.

The performance uses surface based pressures to determine the suitability of each design. This choice allowed for the viscous flow physics in the vicinity of the shockwave to be taken into account within the optimization. The success of the optimal results show that the two-step pressure rise typical of a successful SCB can be achieved with an aero-structurally optimal adaptive SCB. $L / D$ ratios were examined and showed 


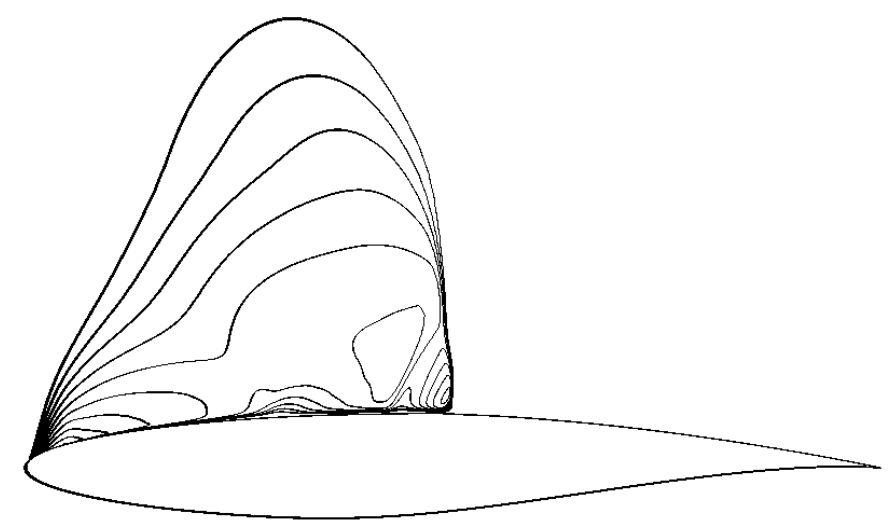

Figure 19. Supersonic Mach Contours for pressure loaded actuated SCB.

small positive change with an increase in actuation height, $(\Delta(L / D)<0.6 \%)$. This was attributed to the development of a low pressure separated flow region downstream of the crest which is omitted from the optimisation if a global performance metric is used. This highlights the difference between designing SCB for global performance and local flow quality. By focussing upon flow quality and incorporating viscous effects, SCB could be designed for stronger shock waves such as those on natural laminar flow airfoils rather than focussing on the global performance of airfoils with weaker shocks.

The small bump actuation heights $(\delta<1.75 \mathrm{~mm})$ are large enough to enforce the bifurcation of the shock yet small enough to negate post-shock separation. The pressure loading increases the ramp angle which increases the oblique shock angle. This has the effect of increasing the triple point height as well as minimising the strength of the rear leg. The effect of the pressure loading enables smaller SCB to exhibit equivalent performance to that of taller SCB. Smearing the pressure across the surface of the SCB not only has a positive aerodynamic effect but reduces the stress concentrations at the points of actuation. This way the design of SCB can utilise the aero-loading to positive effect. It is the pressure loading that has been brought to the fore during this investigation as it has merged the fields of SCB design and aeroelastic panel deformation.

The next challenges that face adaptive SCB both experimentally and numerically will involve the complex aero-structural coupling, this design study has highlighted the effects of pressure loading on adaptive SCB and has touched upon the effects of aeroelastic panel response. The dominant pressure forces surrounding flexible plates have been shown to have little global effect in terms of shock position although close to the wall there is significant effect. These changes are attributed to the flow-plate curvature relationship. The difficulties of extending this to fully time-resolved simulation arise due to the small time-scales required for transonic CFD analyses which drive up the cost of optimization studies. It may be the case that quasi-steady analyses are used to derive aero-structurally optimal geometries which are then subjected to a fully-coupled time-resolved aero-structural analysis.

Further studies could accommodate variable cavity pressures in order to change the geometry response to the shock structure. More actuation or displacement control points could be added to achieve more complex geometries that have become possible due to the consideration of pressure loading.

\section{References}

\footnotetext{
${ }^{1}$ Reneaux, J., "Overview of drag reduction technologies for civil transport aircraft," European Congress on Computational Methods in Applied Sciences and Engineering, 2004.

${ }^{2}$ Ashill, P., Fulker, J. L., and Shires, A., "A Novel Technique for Controllling Shock Strength of Laminar-Flow Aerofoil Sections," First European Forum on Laminar Flow Technology, 1992, pp. 175-183.

${ }^{3}$ Bruce, P. J. K. and Colliss, S. P., "Review of research into shock control bumps," Shock Waves, Oct. 2014.

${ }^{4}$ Ogawa, H., Experimental and Analytical Investigation of Transonic Shock-wave / Boundary-layer Interaction Control with Three-dimensional Bumps, Ph.D. thesis, Clare Hall College, Cambridge University, 2006.

${ }^{5}$ Lee, D., Bugeda, G., Periaux, J., and Onate, E., "Robust active shock control bump design optimisation using hybrid parallel MOGA," Computers \& Fluids, March 2012.
} 
${ }^{6}$ Qin, N., Wong, W. S., and Le Moigne, A., "Three-dimensional contour bumps for transonic wing drag reduction," Proceedings of the Institution of Mechanical Engineers, Part G: Journal of Aerospace Engineering, Vol. 222, No. 5, Aug. 2008, pp. 619-629.

${ }^{7}$ Colliss, S. P., Babinsky, H., Nübler, K., and Lutz, T., "Joint Experimental and Numerical Approach to Three-Dimensional Shock Control Bump Research," AIAA Journal, Vol. 52, No. 2, Feb. 2014, pp. 436-446.

${ }^{8}$ Wadehn, W., Sommerer, A., Lutz, T., Fokin, D., Pritschow, G., and S.Wagner, "Structural Concepts and Aerodynamic Design of Shock Control Bumps," ICAS 2002 Congress, 2002.

${ }^{9}$ Rhodes, O., Optimal Design of Morphing Structures, Phd thesis, Imperial College London, 2012.

${ }^{10}$ Yagiz, B., Kandil, O., and Pehlivanoglu, Y. V., "Drag minimization using active and passive flow control techniques," Aerospace Science and Technology, Vol. 17, No. 1, March 2012, pp. 21-31.

${ }^{11}$ Kutzbach, M., Lutz, T., and Wagner, S., "Investigations on Shock Control Bumps for Infinite Swept Wings," 2nd AIAA Flow Control Conference, June 27-July 1, 2004, Portland, OR, 2004, pp. 1-10.

${ }^{12}$ Ogawa, H., Babinsky, H., Ptzold, M., and Lutz, T., "Shock-Wave/Boundary-Layer Interaction Control Using ThreeDimensional Bumps for Transonic Wings," AIAA Journal, Vol. 46, No. 6, June 2008, pp. 1442-1452.

${ }^{13}$ Colliss, S. P., Babinsky, H., Nübler, K., and Lutz, T., "Vortical structures on three-dimensional shock control bumps," 51st AIAA Aerospace Sciences Meeting including the New Horizons Forum and Aerospace Exposition, No. January, AIAA, Grapevine (Dallas/Ft. Worth Region), Texas, 2013, pp. 1-15.

${ }^{14}$ Sommerer, A., Th., L., and Wagner, S., "Design of Adaptive transonic Airfoils by Means of Numerical Optimization," Proceedings ECCOMAS 2000: European Congress on Computational Methods in Applied Sciences and Engineering, 2000, p. 379.

${ }^{15}$ Eastwood, J. P. and Jarrett, J. P., "Toward Designing with Three-Dimensional Bumps for Lift/Drag Improvement and Buffet Alleviation," AIAA Journal, Vol. 50, No. 12, Dec. 2012, pp. 2882-2898.

${ }^{16}$ AGARD-AR-138, "Experimental Data Base for Computer Program Assessment," Tech. rep., NATO Advisory Group for Aerospace Research and Development.

${ }^{17}$ Cook, P., McDonald, M., and Firmin, M., "Aerofoil RAE 2822 - Pressure Distributions, and Boundary Layer and Wake Measurements," Experimental Data Base for Computer Program Assessment, AGARD Report AR 138., 1979. 